\title{
ETUDE DE LA TRANSMISSION DE LA HOULE A TRAVERS UNE PAROI PERFOREE DU TYPE JARLAN
}

\author{
D. CAMINADE : M. BELORGEY \\ Laboratoire de Mécanique des Fluides \\ Université du Havre. BP 265 - 76055 LE HAVRE CEDEX
}

\begin{abstract}
Within a general research program about the vertical walls with a low reflective power, the subject of this paper is the study of wave transmission through a perforated plate.

Using classic methods of investigation and Laser Doppler Velocimetry, the phenomenon could be explained and some points could be pointed out :

- Three different modes of the general behaviour of the perforated plate could be observed,

- The flow through the holes is a gravity one. The behaviour of the holes is the same whatever their position or their shape are.

- The rules about the drop of level between each side of the plate versus the height of incident wave could be given,

- The transmission through the plate is made without delaying the wave.
\end{abstract}

\section{INTRODUCTION}

Ces dernières années ont vu naitre beaucoup d'innovations en matière d'ouvrages de protection contre la mer et notamment des digues verticales peu réfléchissantes au nombre desquelles on peut compter le système JARLAN.

Si l'on connait le comportement global de cette structure essentiellement constituée d'une paroi perforée côté large, d'une paroi pleine et d'une chambre de dissipation de l'énergie, on connaît beaucoup moins bien le comportement détaillé de cette structure.

Un programme global de recherche financé en partie par le M.R.T. a donc été établi. ll comprend:

A - Au Laboratoire de Mécanique des Fluides de l'Université du Havre:

1/ L'étude d'une paroi perforée seule comportant:

- l'étude du champ des vitesses au voisinage de la paroi 
- l'étude des déphasages temporels entre lamont et l'aval ainsi que l'étude de la dénivelée de la cote de la surface libre

Ces deux études font lobjet du présent exposé et seront complétées ultérieurement par:

- l'étude du champ des pressions sur la paroi (côtés amont et aval)

2/ L'étude d'un caisson schématique comportant une paroi perforée et une paroi pleine portant sur les mêmes items:

- champ des vitesses

- pressions

- niveaux

B - Au Service Central Technique des Ports Maritimes et Voies Navigables (en sous-traitance avec la SOGREAH) :

- l'étude des coefficients de réflexion d'un caisson complet en houle frontale et houle oblique (houle régulière et aléctoire)

- l'étude de stabilité globale de ce caisson dans les mêmes conditions

- l'étude des efforts induits sur un caisson et sur une partie de la parol amont (au large)

- l'étude des niveaux devant le caisson et à l'intérieur de celui-ci

C-Sur le site de l'avant-port de Dieppe

Le Laboratoire de Mécanique des Fluides de l'Université du Havre. grâce à l'aide du Ministère de la Recherche et de la Technologie a entrepris d'expérimenter un caisson de façon à mesurer en vraie grandeur les pressions en différents points du caisson:

- sur la paroi perforée (intérieur eł extérieur)

- sur la paroi opaque (côté chambre)

- sur le fond (côté chambre)

- sous le fond à linterface entre le soubassement et le caisson proprement dit. A cet effet, un caisson a été équipé de 7 capteurs de pression répartis sur laxe médian du caisson.

L'ensemble de ce programme d'études a pour finalité de mieux connaitre les efforts appliqués à une telle structure (que ce soit les efforts globaux ou les efforts appliqués sur les diverses parties d'ouvrages). 


\section{INSTALLATIONS EXPERIMENTALES}

Les études ont été conduites au Laboratoire de Mécanique des Fluides qui est équipé de deux canaux à houle pratiquement identiques du point de vue général mais dont un seul est équipé d'une chaine complète de mesure de vitesses par vélocimétrie laser.

\section{Les canaux à houle}

Les deux canaux à houle sont de conception similaire.

Ils ont une longueur totale de $9 \mathrm{~m}$ pour une largeur de $0,80 \mathrm{~m}$ et une hauteur utile de $0.80 \mathrm{~m}$ compte tenu de la présence du double fond pour une hauteur total de $1 \mathrm{~m}$

La hauteur d'eau utile maximale est de $0.55 \mathrm{~m}$ dans le canal laser et de $0,60 \mathrm{~m}$ environ dans l'autre.

Ils sont constitués de trois parties:

- Un module de génération de la houle avec un batteur plan à mouvement horizontal

- Un module de cissipation de l'énergie de la houle a l'autre extrémité

- Un module central composé de quatre éléments de $1.5 \mathrm{~m}$ de longueur chacun.

Ces éléments sont constitués d'une ossature métallique et de parois en vitre de $10 \mathrm{~mm}$ d'épaisseur.

Le fond est horizontal sauf pour le raccordement entre le canal et le batteur où celui-ci a la forme d'un divergent-convergent, forme destinée à améliorer les caractéristiques de la houle produite.

\section{Les chaines de mesures de la houle}

La mesure de la houle est effectuée grâce à deux sondes de type résistif alimentées en courant alternatif. Ce choix permet d'éliminer les problèmes rencontrés avec les sondes résistives à courant continu (électrolyse et dépôt en particulier).

Ces sondes peuvent être reliées à:

- une table potentiométrique à deux entrées permettant d'enregistrer les variations du niveau de la surface libre,

- un analyseur de spectre à deux voles qui permet un réglage fin de la 
période de la houle et bien entendu de visualiser les harmoniques et les spectres obtenus,

- une mémoire numérique qui permet de stocker les informations sous forme numérique.

Ces chaines de mesures sont à tout à fait classiques et font appel à des techniques éprouvées et fiables.

\section{La chaine de vélocimétrie laser}

La mesure des vitesses à l'intérieur d'un fluide par la méthode de vélocimétrie laser par effet Doppler est utilisée depuis maintenant 8 ans au Laboratoire de Mécanique des Fluides du Havre.

La chaîne utilisée pour les mesures a été récemment modernisée et comprend:

- un laser à deux composantes d'une puissance de $5 \mathrm{~W}$ dont les longueurs d'onde utiles, après sélection des longueurs d'onde donnant le maximum de puissance sont :

$$
\begin{aligned}
& 514,5 \mathrm{~nm} \text { pour le faisceau vert } \\
& 488,0 \mathrm{~nm} \text { pour le faisceau bleu }
\end{aligned}
$$

- un ensemble optique permettant de séparer les faisceaux, de les polariser et de régler leur chemin optique à un écartement de $50 \mathrm{~mm}$.

- deux cellules de Bragg dont le meilleur réglage semble être de 100 $\mathrm{KHZ}$

- un ensemble de réception du signal comportant notamment:

* 2 photo-multiplicatuers

* 1 mémoire numérique

* 1 Trigger

* 2 compteurs d'impulsions

* 1 oscilloscope pour visualiser les signaux (permettant ainsi le réglage de l'optique de réception)

- un ensemble de traitement des signaux permettant, à partir des données brutes, et grâce à un logiciel spécifique développé au LMFH dit programme LDV, de calculer pour chaque point repéré par ses coordonnées $x$, y (les notations sont celles utilisées par le programme) :

* la vitesse horizontale U

* la vitesse verticale $V$

* les dérivées temporelles DU et DV

* la covariance de U et $V$. UV 
Cet ensemble de données étant appelé point de mesure.

Le réglage de l'ensemble de la chaine de vélocimétrie laser fait appel à de très nombreux paramètres.

On peut visualiser à l'écran les résultats des mesures des vitesses comme le montrent à titre d'exemple les figures ci-après:

- Fig. 1 = vitesses obtenues à proximité d'un trou de la plaque pour $\mathrm{T}=7,2 \mathrm{~s}$ et $\mathrm{H}=2.7 \mathrm{~m}$

- Fig. 2 = vitesses obtenues à une distance nature de $3 \mathrm{~m}$ de celle$\mathrm{ci}$

Les échelles de vitesses sont dans un rapport de 3 à 10.
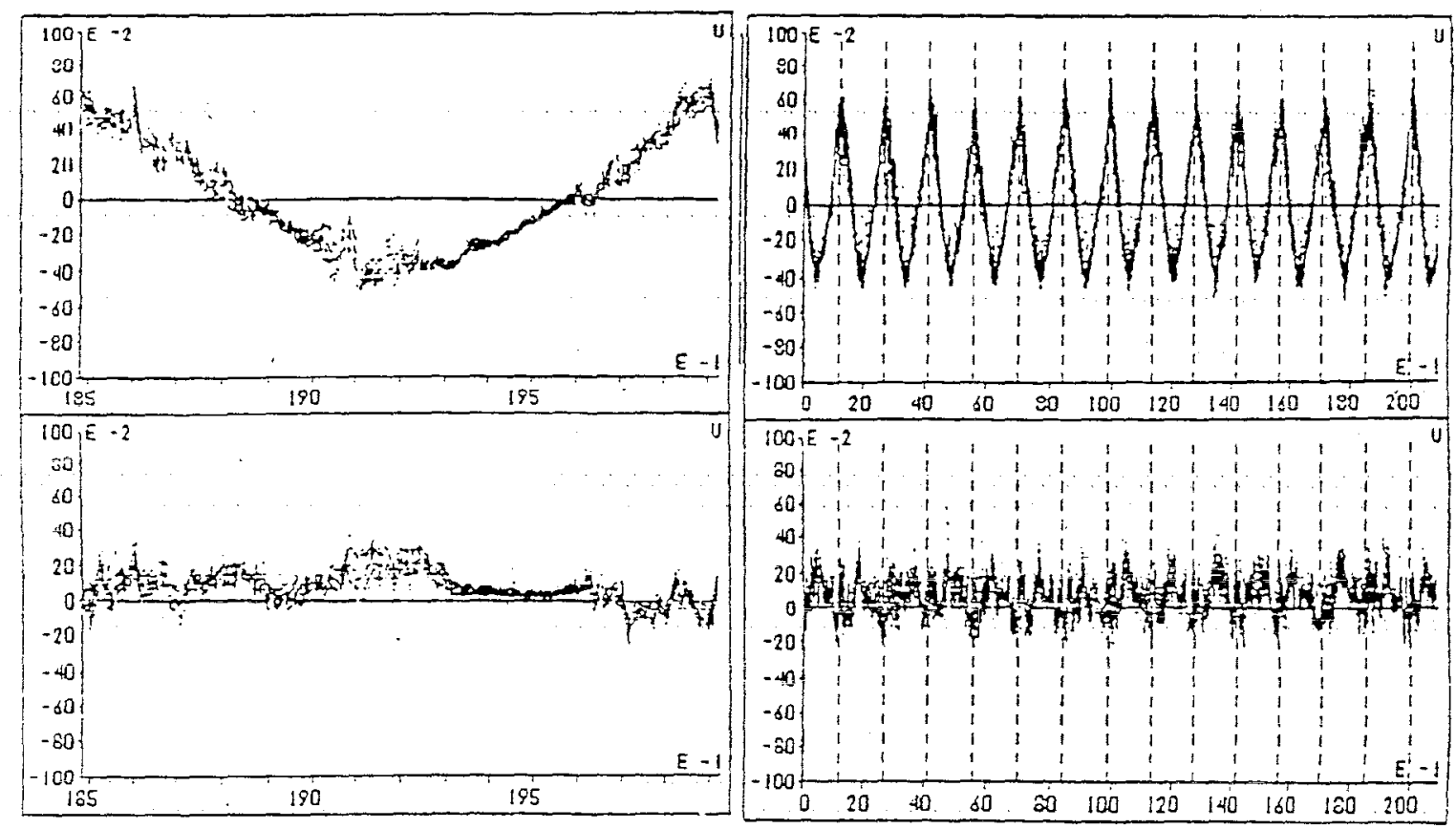

Fig. 1: Vitesses obtenues à proximité d'un trou de la plaque pour

$$
T=7.2 \mathrm{~s} \text { et } \mathrm{H}=2.7 \mathrm{~m}
$$



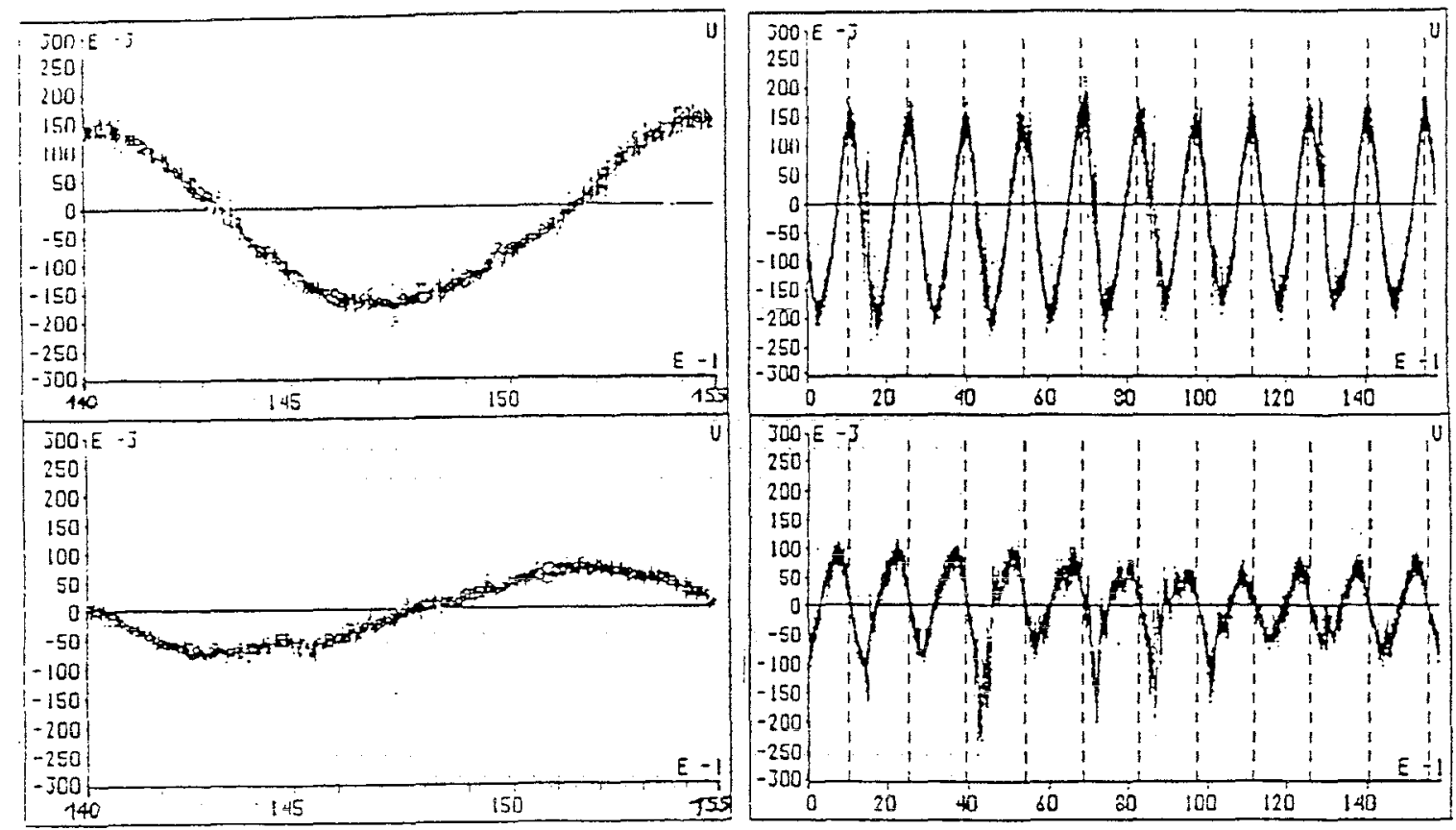

Fig.2: Vitesses obtenues à une distance nature de $3 \mathrm{~m}$ de celle-ci

\section{LES RESULTATS}

Les essais conduits à l'échelle du 1/25 par rapport à la nature ont permis de mieux appréhender les phénomènes qui régissent le mode de transmission de la houle à travers la paroi seule.

Pour une centaine de cas d'essais correspondant chacun à un creux et une période de la houle incidente, on a analysé le comportement hydraulique de la plaque.

\section{Mode de fonctionnement}

Les études globales menées par visualisation et par mesure de la cote de la surface libre ont permis de définir 3 modes de fonctionnement de la paroi:

- le premier ou la plaque laisse passer pratiquement toute l'énergie de la houle jusqu'à une cambrure quel'on peut estimer à $2,5 \%$,

- un mode de fonctionnement intermédiaire où, bien que la plaque n'absorbe que très peu d'énergie, on constate une différence de niveau significatif entre les deux côtés de la plaque.

- enfin un mode de fonctionnement qui se rapproche beaucoup de celui des digues verticales, malgré un transfeit d'énergie à travers celle-ci. 
Le creux de la houle transmise H† peut être exprimé pour les diverses valeurs de la période en fonction de la houle incidente Hi par les formules:

\begin{tabular}{|c|c|}
\hline PERIODE & CREUX TRANSMIS \\
\hline $4.9 \mathrm{~s}$ & $\mathrm{Ht}=\mathrm{Hi}-0.114 \mathrm{Hi}^{1,954}$ \\
$6.1 \mathrm{~s}$ & $\mathrm{Ht}=\mathrm{Hi}-0.122 \mathrm{Hi}^{1.481}$ \\
$7.2 \mathrm{~s}$ & $\mathrm{Ht}=\mathrm{Hi}-0.119 \mathrm{Hi}^{1.408}$ \\
$9.1 \mathrm{~s}$ & $\mathrm{Ht}=\mathrm{Hi}-0.060 \mathrm{Hi}^{1.951}$ \\
\hline
\end{tabular}

qui montrent une similitude pour les périodes de 6 à $7 \mathrm{~s}$ pour lesquelies on peut écrire:

$$
H t=H i-0.12 H i 3 / 2
$$

\section{Différence de nivequ entre les deux côtés de la plaque}

Les mesures effectuées grâce à la technique de vélocimétrie laser Doppler ont permis dans deux cas $T=7,2 \mathrm{~s}, \mathrm{H}=1.4 \mathrm{~m}$ et $\mathrm{H}=2.7 \mathrm{~m}$ d'établir que le mode de fonctionnement général de la plaque est celui d'un écoulement de type gravitaire à travers les orifices, l'écoulement étant généré par la différence de niveau entre le côté houle et le côté abrité.

Cette différence de niveau est fonction:

des caractéristiques de la houle qui compte tenu de l'épaisseur de l'obstacle génère une différence de niveau. du coefficient de réflexion de la plaque qui en augmentant lagitation devant celle-ci. augmente la différence de niveau et permet aussi un meilleur tranisfert de l'énergie.

du déficit de niveau et d'agitation constaté à l'arrière de la plaque qui contribue à lame montante à un meilleur transfert de la houle à travers l'obstacle.

Elle peut être traduite par les formules simples ci-après:

\begin{tabular}{|c|c|c|}
\hline PERIODE & CRETE & CREUX \\
\hline $4.9 \mathrm{~s}$ & $0.4 \mathrm{Hi}$ & $-0.38 \mathrm{Hi}$ \\
$6.1 \mathrm{~s}$ & $0.38 \mathrm{Hi}$ & $-0.30 \mathrm{Hi}$ \\
$7.2 \mathrm{~s}$ & $0.35 \mathrm{Hi}$ & $-0.35 \mathrm{Hi}$ \\
$\mathrm{Hi} \leq 3 \mathrm{~m}$ & $0.3 \mathrm{Hi}+0.005 \mathrm{Hi}^{3.4}$ & $-0.3 \mathrm{Hi}$ \\
Ensemble & $0.15 \mathrm{Hi}^{1 / 475}$ & $-0.05 \mathrm{Hi}$ \\
$9.1 \mathrm{~s}$ & & \\
\hline
\end{tabular}


Pour l'ensemble des périodes, on peut donner en première approximation les formules très simples suivantes:

$$
\begin{aligned}
& \text { Crête } 0.35 \mathrm{Hi}<\Delta \mathrm{H}<0,40 \mathrm{Hi} \\
& \text { Creux } 0.30 \mathrm{Hi}<\Delta \mathrm{H}<0,40 \mathrm{Hi}
\end{aligned}
$$

formules quil sont conservatrices pour $\mathrm{T}=9,1 \mathrm{~s}$ ef $\mathrm{Hi}<4 \mathrm{~m}$.

Pour les houles fortes, la mesure des seuls niveaux n'est pas suffisante et seul la mesure des pressions permettra de donner des règles de dimensionnement : en effet, les surélévations locales mesurées à l'amont (dues à l'ascension verticale des lames) ne devraient pas générer une augmentation de pression correspondante, sur l'ensemble de la verticale car l'épaisseur de cette surélévation est trop faible.

II s'avère donc nécessaire de compléter les mesures réalisées par des mesures de pression, pour mieux connaitre l'effet des lames les plus fortes sur de telles structures.

\section{Type d'écoulement à travers la plaque}

Du fait que le transfert d'énergie à travers la plaque se fait essentiellement par des jets alternés pour les orifices situés constamment dans l'eau. on peut dire que :

- conformément aux résultats obtenus par d'autres études réalisées avec des trous rectangulaires, la forme des trous n'a que peu d'importance sur le mode de fonctionnement de la plaque.

l'importance du transfert d'énergie est quasiment indépendante de la position des trous. Ainsi et contrairement à une opinion que nous avons rencontrée, les trous situés près du fond permettent un transfert d'énergie du même ordre de grandeur que ceux situés au-dessus et sont donc aussi importants dans la conception de la structure. La différence de vitesse constatée sur une même verticale est plutôt due aux courants de houle qu'à la position des trous eux-mêmes. 

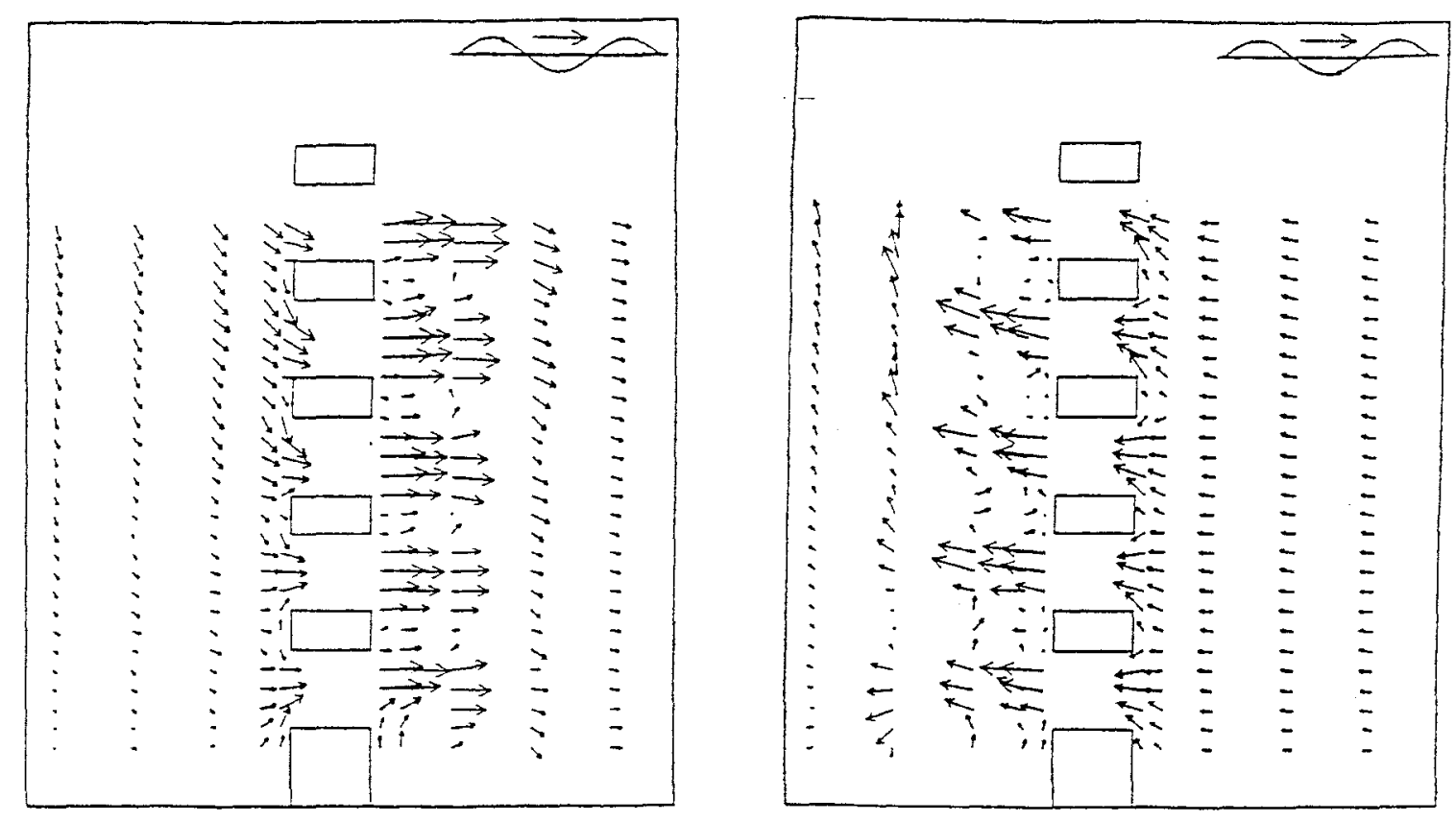

Fig. 3: Champ des vitesses pour $\mathrm{T}=7.2 \mathrm{~s} . \mathrm{H}=2.7 \mathrm{~m}$, phases $30^{\circ}$ et $210^{\circ}$

\section{Déphasage Amont-Aval}

Les investigations que nous avons menées ne nous ont pas permis de mettre en évidence un déphasage temporel dú au passage de la plaque : la houle se propage donc sans être freinée.

Ceciest confirmé :

- d'une part par lexamen des roses des vitesses pour lesquelles on constate un décalage des points singuliers tels que $U$ max ou $U=0$ correspondant à la célérité de la houle.

- dautre part par l'examen des champs des vitesses. A titre d'exemple la figure 4 montre que linversion de vitesses horizontales pour les profils extrêmes a lieu à des instants dont le décalage $\left(30^{\circ}\right)$ correspond à la célérité de la houle. 


\section{Phénomène de filtre}

Nous avons pu constater que les perturbations étaient limitées au voisinage immédiat de la plaque : $1 \mathrm{~m}$ à $1.5 \mathrm{~m}$, nature suivant le creux de la houle $(1,4 \mathrm{~m}$ ou $2.7 \mathrm{~m})$.

La plaque permet de générer à l'aval une houle plus régulière que celle constatée en amont: l'énergie qui traverse la plaque est remaniée et remélangée pour donner naissance à une nouvelle houle homogène: il y a recomposition de la houle au passage de la plaque.

Ceci explique en partie le déficit d'agitation constaté juste derrière l'obstacle car il faut une certaine distance à la houle pour se reformer et adopter un profil de vitesse décroissant de haut en bas sur une même verticale alors que comme nous l'avons dit, les jets sont quasiment identiques du haut en bas.

La forte mise en vitesse des particules fluides au passage de la plaque génère vraisemblablement un phénomène de VENTURl et contribue ainsi au déficit constaté.
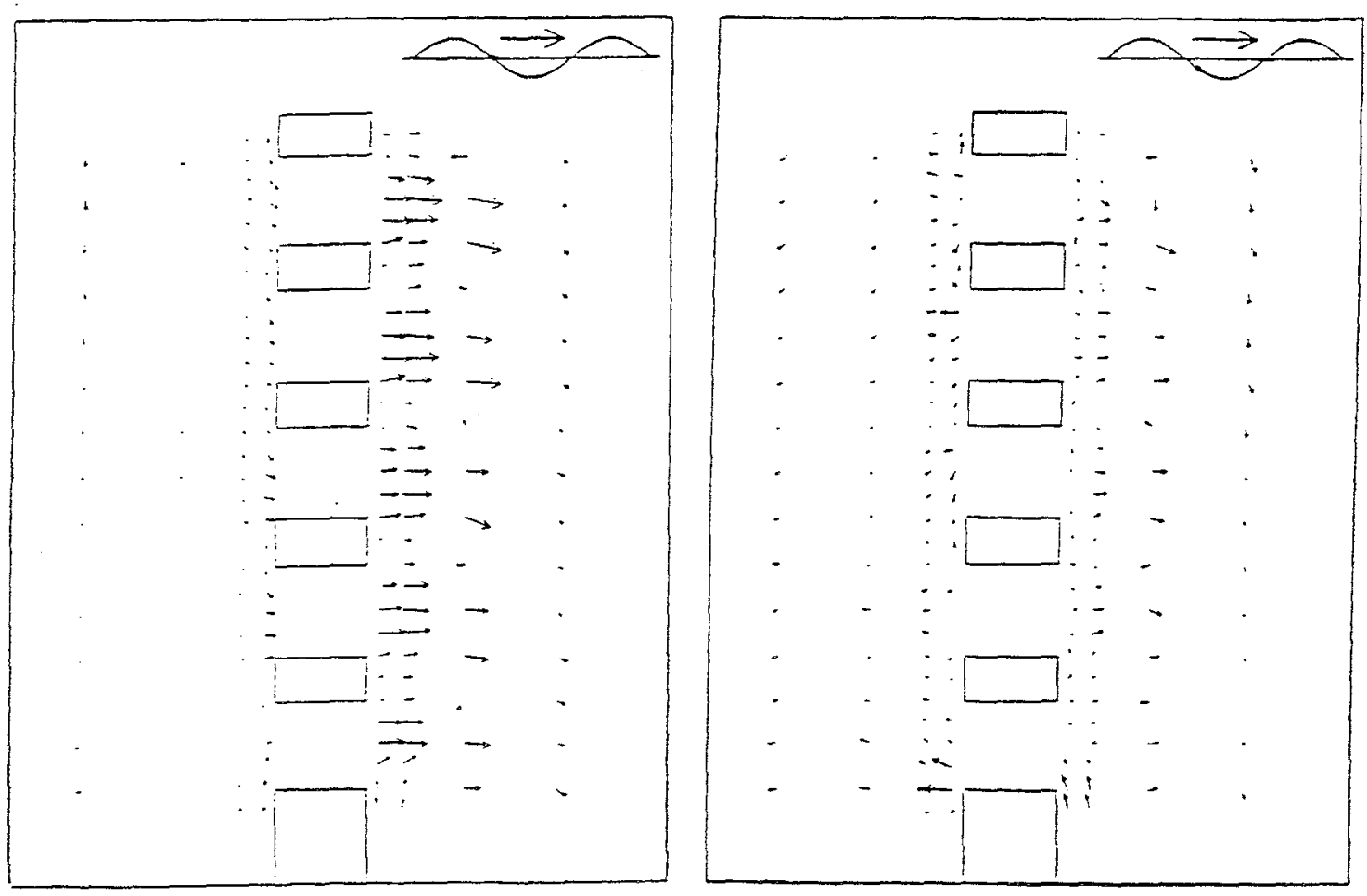

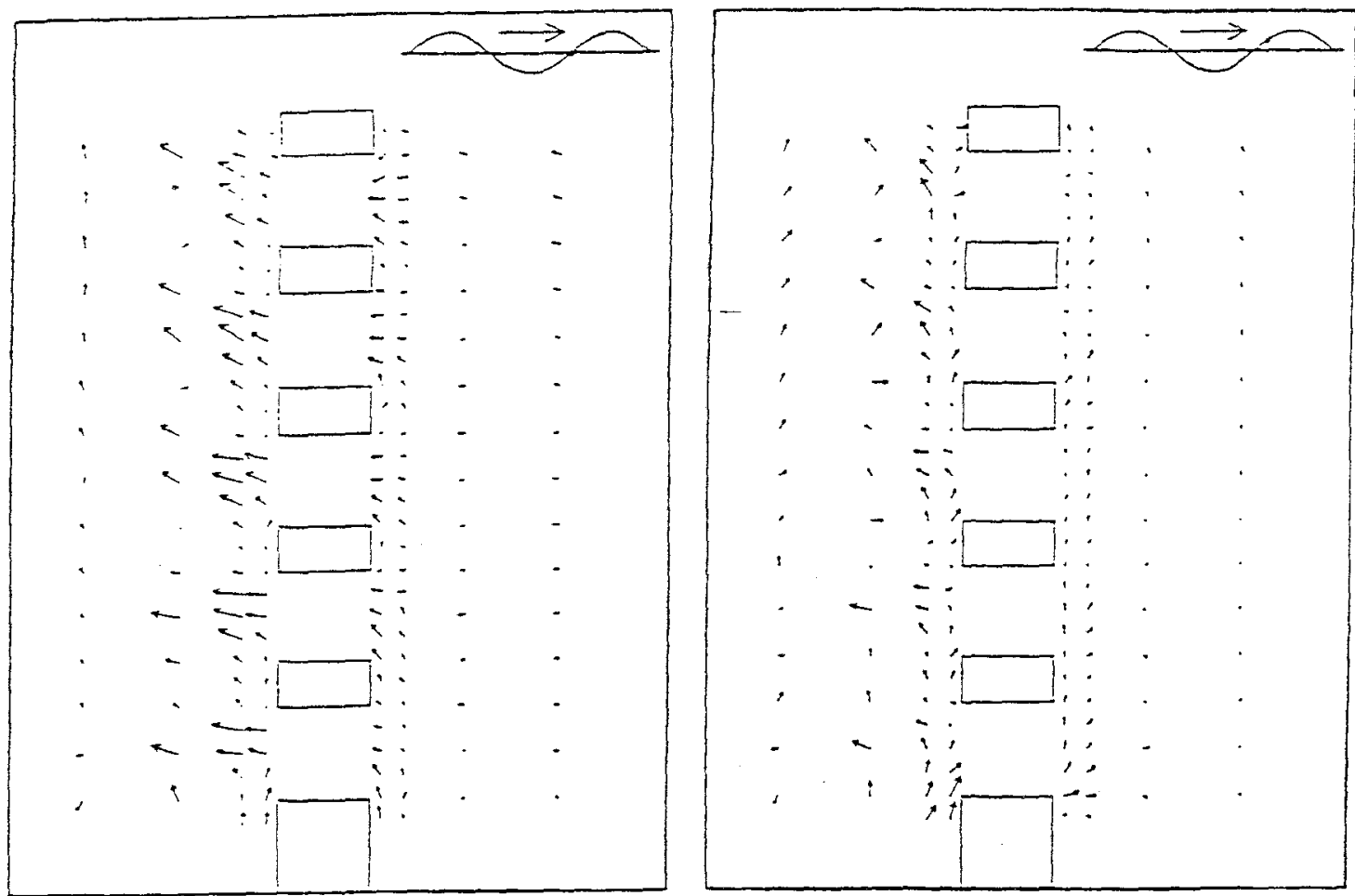

Inversion des vitesses horizontales à vague montante

(phases $270^{\circ}$ et $300^{\circ}$ )

Fig. 4: Champ des vitesses pour $\mathrm{T}=7,2$ ef $\mathrm{H}=1,4 \mathrm{~m}$

\section{CONCLUSION}

L'ensemble de cette étude a permis:

I/ de bien comprendre le mode de fonctionnement d'une paroi perforée et de caractériser la transmission de l'énergie de la houle. Les mouvements de la masse fluide observés soit visuellement, soit en ananlysant les vitesses mesurées par VDL nous permettenł de les comparer à ceux d'un piston hydraulique déformable: la plaque réagit pour sa partie immergée comme un batteur à houle souple.

2/ d'acquérir suffisamment de données globales (niveaux de la surface libre) ou ponctuelles (vitesses au voisinage de la plaque ou à proximité d'un trou) pour pouvoir envisager une approche numérique du problème.

3/ de guider une réflexion portant sur l'amélioration de ce type d'ouvrages

4/ de préparer les futures campagnes de mesures sur site ou en laboratoire. 


\section{REFERENCES BIBLIOGRAPHIQUES}

(1) M. BELORGEY - J. LE BAS

"Etude par vélocimétrie Doppler Laser du champ des vitesses instantanées dans un canal à houle produite en laboratoire" La Houille Blanche, $n^{\circ} 5,1984$.

(2) R. BOIVIN

"Comments on Vertical Breakwaters with low coefficients of reflexion the Dock and Harbour Authority, n'524, June 1964

(3) D. CAMINADE

Interaction houle-structures. Etude de la transmission de la houle à travers une paroi perforée (type Jarian).

Thèse Doctorat de l'Université du Havre. Février 1992.

(4) G.E. JARLAN

The application of acoustic theory to the reflective properties of coastal engineering structures.

National Research Council of Canada, Ottawa, April 1965.

(5) LABORATOIRE DE MECANIQUE DES FLUIDES/UNIVERSITE DU HAVRE Etude sur modèle réduit des efforts exercés par la houle sur les parois des caissons Jarlan de la digue du port de Dieppe.

Juillet 1987.

(6) LABORATOIRE CENTRAL D'HYDRAULIQUE DE FRANCE (LCHF) Réception des grands pétroliers - Terminal en Baie de Seine Ełude des caissons perforés, 1970-1971.

(7) A. MERRIEN lle d'Ouessant. Etude et construction de la digue du Stiff Stage IPER "Choix, conception, surveillance et entretien des digues", Le Havre, 21-23 avril 1986.

(8) A. MERRIEN Etude et réalisation de la digue du stiff à Ouessant Annales des Ponts et Chaussées, ler trimestre 1987. 